\title{
Circumstellar Chemistry of AGB Winds
}

\author{
A. E. Glassgold \\ Physics Department, New York University, 4 Washington Place, \\ New York, NY 10003, USA
}

\begin{abstract}
.
Carbon-rich AGB stars are an excellent place to study circumstellar chemistry. The best case is IRC +10216 , where observations at optical, infrared, and radio wavelengths have led to the discovery of more than 50 molecules. When the detected species are grouped in chemically related families, the observed spatial distributions and abundances can be understood with a photochemical model. Most of the species are either progenitors that arise in the photosphere or inner envelope, or are unsaturated radicals and hydrocarbon chains photochemically produced in the outer envelope. The few saturated molecules with a single heavy atom are probably produced on grain surfaces. Inside the ionization radius of atomic carbon, the circumstellar envelope of IRC +10216 is weakly ionized by suprathermal particles. The $\mathrm{HCO}^{+}$detected and mapped in the $J=1-0$ line by the IRAM telescopes can be interpreted as arising from either partially-excluded galactic cosmic rays or $\beta$-particles emitted by radioactive nuclei dredged up from the $\mathrm{H}$-burning zone into the wind. An estimate of the ionization rate due to ${ }^{26} \mathrm{Al}$ based on the ${ }^{26} \mathrm{Al} /{ }^{27} \mathrm{Al}$ ratio of "mainstream" pre-solar grains is the same order of magnitude as determined from the measured abundance of $\mathrm{HCO}^{+}$in $\mathrm{IRC}+10216$.
\end{abstract}

\section{Introduction}

High-density winds emerge from stars at various stages of evolution. For low and intermediate mass stars, this occurs when they form and just before they die, e.g., as AGB stars with mass-loss rates in the range $10^{-4}-10^{-5} M_{\odot} \mathrm{yr}^{-1}$. The rapid decrease with distance of the density of these winds is accompanied by strong adiabatic and line cooling ${ }^{1}$. The combination of decreasing wind density and temperature means that the abundances close to the star tend to get frozen into the wind, to be carried out to large distances where they can be observed by radio astronomy. This conclusion is dramatically altered when the effects of external agents such as interstellar UV radiation are considered. A main contention of this review is that most of the molecular complexity observed in AGB winds is induced by the interstellar radiation field. We also show that

\footnotetext{
${ }^{1}$ In the absence of heating, the temperature would fall below that of the cosmic background radiation.
} 
live radionuclides dredged up from the interior of an AGB star can affect the ionization of the outer circumstellar envelope (CSE) and be observable by radio observations of molecular ions. An earlier but more complete review of wind chemistry is also available (Glassgold 1996a).

Nuclear burning in an AGB star occurs in a double shell outside of a burntout core. Convection currents dredge up newly processed material through the huge stellar envelope to the photosphere. Stellar dynamical processes, possibly pulsational shocks (see the review by Willson et al. 1997), drive the outer layers of the star and distend the atmosphere, producing physical conditions favorable for the formation of dust. Once formed, the dust can be driven out by radiation pressure and drag the gas along to generate the wind. The complex and poorly understood processes that form dust and produce the wind occur in a "transition region" between radial distances $\sim 10^{14}-10^{15} \mathrm{~cm}$. A detailed knowledge of the dynamics of this region is currently unavailable but is crucial for determining its physical and chemical properties. Although interferometry is now beginning to provide images on this scale, most of the chemical information that we have on the transition region is based on high spectral resolution infrared spectroscopy (e.g., Keady \& Ridgway 1993).

Once dust formation and wind generation have ceased, the dust and gas (with depleted abundances) expand at constant velocities. At first the dust and gas interact but they eventually become uncoupled as the density and the temperature decrease. The gas-phase abundances become fixed at a new quasi-LTE equilibrium (see Sect. 2.5). If nothing else occurred, there would be no further chemical evolution. What happens, however, is that the wind becomes exposed to interstellar UV radiation, and all of the molecular species are broken down by photodissociation into atoms, which are then photoionized (if their ionization potentials $I P<13.6 \mathrm{eV}$ ). Before being destroyed, however, the radicals produced by molecular photodissociation can synthesize other (possibly more complex) molecules, especially in the case of C-rich CSEs. The only complexes that survive from the inner part of the CSE are solids that serve as the seeds for the development of interstellar dust.

Thus the circumstellar matter that leaves the cool surface of an AGB star is profoundly altered by its passage through the inner transition region, where dust is formed, and by photodissociation at intermediate distances. The latter exposure involves both molecular synthesis and destruction, and produces shells of radicals and other compounds, including molecular ions. The physical conditions in the shells are similar to those in interstellar molecular cloud cores, and are observable in a wide range of molecular rotational lines. In the next section we review the extensive observations of the nearby C-rich AGB star, IRC +10216. Focusing on this well-studied object allows detailed theoretical interpretations to be made. This envelope is approximately spherical at intermediate to large distances, as is the case for most AGB stars (Guélin \& Lucas, personal communication). However, the detection of clumps and other substructure in the photochemical shells clearly indicates that the envelope is inhomogeneous. Nonetheless, the application of a very simple photochemical model based on a spherical and homogeneous CSE proves to be quite illuminating. 


\section{Chemical observations of IRC +10216}

\subsection{Astrophysical properties}

High-resolution radio line spectra of IRC +10216 conform closely to expectations for an expanding envelope and permit an accurate determination of the maximum expansion velocity, $V=14 \mathrm{~km} \mathrm{~s}^{-1}$. The measurement and mapping of $\mathrm{CO}$ lines, together with the distance $d$, yield the hydrogen nucleus mass-loss rate $\dot{M}$ and the $\mathrm{CO}$ abundance $x(\mathrm{CO})$. For $d=200 \mathrm{pc}$, values accurate to $50 \%$ are $\dot{M}_{\mathrm{H}}=2 \times 10^{-5} M_{\odot} \mathrm{yr}^{-1}$ and $x(\mathrm{CO})=4.5 \times 10^{-4}$. Considerations on the bolometric luminosity (Zuckerman et al. 1986) indicate a smaller distance, possibly $d \approx 150$ pc (see, e.g., Groenewegen et al. 1998).

The abundances of the two next most abundant species after $\mathrm{CO}, \mathrm{C}_{2} \mathrm{H}_{2}$ and $\mathrm{HCN}$, can be determined directly by IR absorption and indirectly from the $\mathrm{mm}$ emission of the photodissociation products, $\mathrm{C}_{2} \mathrm{H}$ and $\mathrm{CN}$. The two methods agree to within the errors (probably no better than a factor of 2), and yield $x\left(\mathrm{C}_{2} \mathrm{H}_{2}\right)=4.0 \times 10^{-5}$ and $x(\mathrm{HCN})=2.0 \times 10^{-5}$. All of the abundances quoted so far are relative to total hydrogen nuclei and refer to the part of the CSE that is outside of the region where dust is formed and inside the photodissociation zone. These abundances should be smaller than photospheric due to inclusion of certain elements into or onto dust grains. The most likely example in a C-rich environment is the $\mathrm{C}$ in $\mathrm{C}_{2} \mathrm{H}_{2}$, generally considered the molecular progenitor of carbonaceous dust. Since IR spectroscopy measures $\mathrm{C}_{2} \mathrm{H}_{2}$ just above the photosphere, and radio astronomy measures $\mathrm{C}_{2} \mathrm{H}$ in the outer envelope, a comparison between the two could yield the amount of $\mathrm{C}$ in dust. However, the observations are not yet accurate enough for this purpose.

\subsection{The photochemical model}

The underlying ideas of the model are closely related to the Haser-type model for cometary comae and to photodissociation models that were developed for interstellar clouds in the 60's and 70's. The early development of the photochemical model for CSEs is reviewed by Glassgold (1996a) with full literature references.

The essence of the photochemical model is the processing by UV radiation of species that enter the inner envelope from the transition region. The main processing of carbon can be represented by:

$$
\mathrm{C}_{2} \mathrm{H}_{2} \& \mathrm{HCN} \rightarrow \mathrm{C}_{2} \mathrm{H} \& \mathrm{CN} \rightarrow \text { hydrocarbons } \rightarrow \mathrm{C} \rightarrow \mathrm{C}^{+},
$$

where the first and last stages are radiative. The hydrocarbon synthesis is mainly accomplished by neutral reactions starting with the radicals $\mathrm{C}_{2} \mathrm{H} \& \mathrm{CN}$ (see Sect. 2.4). Fig. 2 of Glassgold (1996a) shows the spatial distribution of the most abundant $\mathrm{C}$ species, particularly how the radicals and their products are distributed in shells. This prediction of the first photochemical calculations has been confirmed by maps made with mm-wave interferometers (e.g., Lucas 1994, Lucas \& Guélin 1998). We are thus led to a simple classification of circumstellar molecules according to their spatial distribution as (1) centrally peaked species coming out of the inner transition region or (2) shell molecules that are synthe- 
sized in the outer CSE. Of course there are some exceptions, such as molecular ions and others to be mentioned in the next section.

\subsection{Observed species}

In Table 1, the more than 50 species detected in IRC +10216 are grouped into families (columns) and listed according to approximate measured abundances. The last column is a mixed bag of heavy-element molecules (excluding $\mathrm{S}$ and $\mathrm{Si}$, columns $7 \& 8$ ) that may not be all related. Except for these heavy elements, whose study is really only getting started, we have a reasonably good understanding of each of the families in Table 1.

Table 1. Species detected in IRC $+10216^{1,2}$

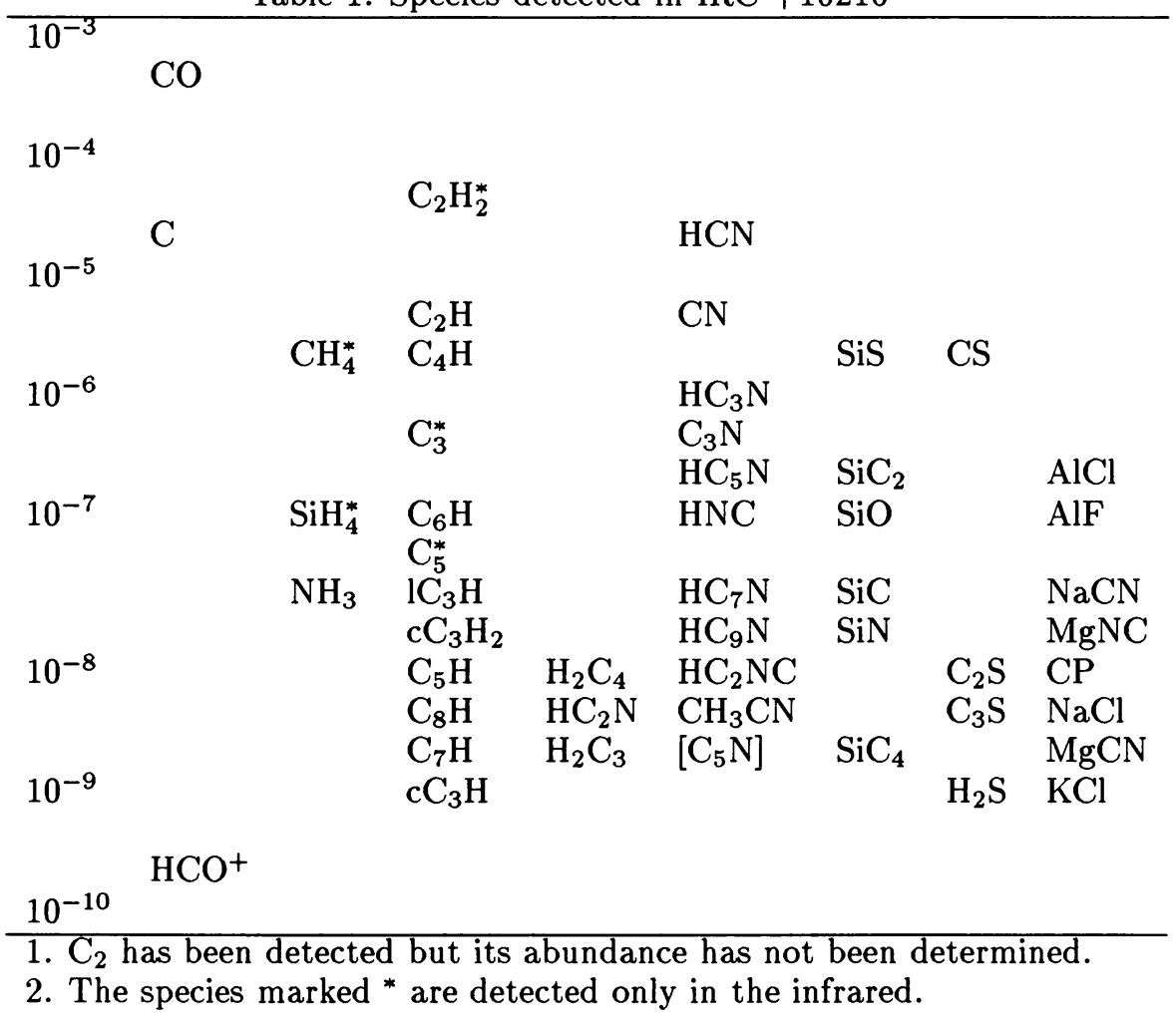

The second column of Table 1 lists $\mathrm{CO}$ and its observed C-bearing descendants, atomic $\mathrm{C}$ and $\mathrm{HCO}^{+}$. Attempts to detect the $158 \mu \mathrm{m}$ line of $\mathrm{C}^{+}$with ISO have not been successful. The $\mathrm{CO}$ family is not important chemically because $\mathrm{CO}$ photodissociation occurs at rather large distances due to line self-shielding (Mamon et al. 1988) where the density and the abundances of potential reactants are low. We will discuss the very weak production of $\mathrm{HCO}^{+}$in Sect. 3 . The 4th and 6th columns contain the combined effects of the photodissociation and radical chemistry associated with $\mathrm{C}_{2} \mathrm{H}_{2}$ and $\mathrm{HCN}$. The 5 th column contains some of the isomers (carbenes) obtained in the process. These three families illustrate the high level of molecular complexity observed in IRC +10216 , characterized by unsaturated carbon chains. The 3 rd column contains the saturated 
molecules, $\mathrm{CH}_{4}, \mathrm{NH}_{3}$, and $\mathrm{SiH}_{4}$. Near-IR spectroscopy indicates that they are not produced in the photosphere, but further out in the inner CSE, where the timescale for gas atoms to interact with dust is still short compared to the expansion time, $r / V$. These molecules are probably desorbed by the energy released in formation.

The 7 th and 8 th columns list the $10 \mathrm{Si}$ - and $\mathrm{S}$-bearing molecules that have been observed in the radio. The LTE calculations of Lodders and Fegley (1995) suggest that $\mathrm{SiS}$ and atomic $\mathrm{Si}$ are the main progenitors, and this is supported by the IRAM maps of SiS and CS, which are concentrated towards the star. The SiS distribution actually shows a rapid decrease very close in, which has been interpreted in terms of depletion of SiS onto grains (e.g., Glassgold \& Mamon 1993). In any case, substantial amounts of SiS and CS reach the photodissociation region, where they are transformed into $\mathrm{Si}$ and $\mathrm{S}$ atoms and ions. The latter then engage in ion-molecule and neutral reactions to produce the rest of the species listed in columns $7 \& 8$. Maps of $\mathrm{SiC}_{2}$ (Gensheimer et al. 1995) indicate that, although its abundance peaks in the outer envelope, some of it is produced in the inner envelope. This supports the idea that $\mathrm{SiC}_{2}$ is also a progenitor molecule (Howe \& Millar 1990). It would be particularly interesting to measure $\mathrm{SiC}_{2}$ in the near-IR to learn more about its distribution near the star. MacKay \& Charnley (1998) have suggested that it is also produced on grains.

In summary, the molecular observations of IRC +10216 can be understood qualitatively in terms of the photochemical model. We do not attempt to present a full case for this conclusion in this short report. However, it is important to emphasize that mapping a species is crucial in determining whether it is centrally peaked or peaked in a shell far from the star. For theoretical modeling, we refer the reader to the literature, e.g., Cherchneff at al. (1993a, henceforth CGM), Cherchneff at al. (1993b), Millar \& Herbst (1994), and Glassgold (1996a), as well as to the discussion of modeling assumptions in Sect. 2.5.

\subsection{Dominance of neutral reactions}

In light of the qualitative success of the photochemical model, it is important to identify the main mechanisms for synthesis in the outer CSE. We use the simplest polyacetylene, $\mathrm{HC}_{3} \mathrm{~N}$, as an example and discuss the relative importance of ionmolecule and neutral radical reactions.

Synthesis by ion-molecule reactions

$$
\begin{gathered}
\mathrm{h} \nu+\mathrm{C}_{2} \mathrm{H}_{2} \rightarrow \mathrm{C}_{2} \mathrm{H}_{2}^{+}+\mathrm{e} \\
\mathrm{C}_{2} \mathrm{H}_{2}^{+}+\mathrm{HCN} \rightarrow \mathrm{H}_{2} \mathrm{C}_{3} \mathrm{~N}^{+}+\mathrm{H}
\end{gathered}
$$

followed by e $+\mathrm{H}_{2} \mathrm{C}_{3} \mathrm{~N}^{+} \rightarrow \mathrm{HC}_{3} \mathrm{~N}+\mathrm{H}$.

Synthesis by neutral reactions

$$
\begin{gathered}
\mathrm{h} \nu+\mathrm{HCN} \rightarrow \mathrm{CN}+\mathrm{H} \\
\mathrm{CN}+\mathrm{C}_{2} \mathrm{H}_{2} \rightarrow \mathrm{HC}_{3} \mathrm{~N}+\mathrm{H}
\end{gathered}
$$

In both cases, the first step is a photoreaction that produces an ion or radical. The measured reaction rate coefficients for the next steps, which produce the 
protonated or the neutral form of $\mathrm{HC}_{3} \mathrm{~N}$, are about the same, $\approx 5 \times 10^{-10} \mathrm{~cm}^{3} \mathrm{~s}^{-1}$ at $30 \mathrm{~K}$ (the temperature in the photochemical shell). Recombination of protonated $\mathrm{HC}_{3} \mathrm{~N}$ is very fast. Thus neutral reactions dominate because the abundance of $C N$ is between 100 and 1000 times larger than that of $\mathrm{C}_{2} \mathrm{H}_{2}^{+}$.

\subsection{Modeling issues}

Although we omit detailed discussion of the theory, the qualitative success of the photochemical model suggests that we examine the basic assumptions of the simple version used so far.

Dynamical assumptions The wind density is almost always prescribed by assuming that the flow is steady, spherically symmetric, and locally homogeneous (unclumped). Although there is observational evidence to the contrary, CO observations that sample the average density of the wind in IRC +10216 indicate approximate spherical symmetry in the region where most molecules are observed.

Chemistry In accord with the observations summarized in Table 1, the modeling of an AGB wind requires the chemistry of a diverse array of elements: $\mathrm{C}, \mathrm{N}, \mathrm{O}, \mathrm{Si}, \mathrm{S}, \mathrm{Mg}$, and other heavy elements. Both neutral and ion-molecule reactions have to be specified, generally at low temperatures, e.g., $T \approx 30 \mathrm{~K}$ in the photochemical shell. Most of the required reaction rates have not been measured, and very few at the appropriate temperature. Similarly most of the photorates have not been measured or calculated theoretically, and very little is known about dust surface chemistry. This situation obviously requires considerable discretion on the part of both modelers and those who use the theory to interpret observations.

Initial conditions The initial abundance of the main progenitors entering the outer CSE from the transition region were originally considered as unknown phenomenological parameters. In time, the initial abundances of $\mathrm{CO}, \mathrm{C}_{2} \mathrm{H}_{2}$, and $\mathrm{HCN}$ determined by the analysis of $\mathrm{mm}$ observations of the outer envelope have come into reasonable agreement with those obtained from near infrared spectroscopy (Keady \& Ridgway 1993). The accuracy of both the radio and infrared measurements are not yet good enough to definitively show any significant difference between the inner and the outer envelope. The envelope abundances of the major species probably do not differ much from photospheric abundances. The calculations of Willacy \& Cherchneff (1997) support this position. They show that, when such molecules are destroyed by pulsational shocks, they are reformed as the gas cools to roughly the original abundances. The calculations of Lodders \& Fegley (1995) also indicate that an effective LTE holds in the inner regions of AGB envelopes.

\section{Molecular ions}

Despite the dominance of neutral reactions in the synthesis of complex hydrocarbons, ion-molecule reactions and molecular ions are still important in circumstellar chemistry (Glassgold, Lucas \& Omont 1986). Dissociative recombination 
of molecular ions can produce isomeric as well as normal molecular forms, e.g., $\mathrm{e}+\mathrm{HCNH}^{+} \rightarrow \mathrm{HNC}$ as well as $\mathrm{HCN}$ and $\mathrm{H}+\mathrm{CN}$, as discussed further by CGM. Another example is the production of the ring molecule $\mathrm{C}_{3} \mathrm{H}_{2}$ in the dissociative recombination of $\mathrm{C}_{3} \mathrm{H}_{3}^{+}$. Of course, the most basic goal of observing molecular ions is to measure the electron fraction.

Table 2. Most Abundant Molecular Ions

\begin{tabular}{ccccc}
\hline Molecule & $I P(\mathrm{eV})$ & $p a(\mathrm{eV})$ & Ion & Production \\
\hline $\mathrm{H}_{2}$ & 15.4 & 4.39 & $\mathrm{H}_{3}^{+}$ & $\mathrm{CR}$ \\
$\mathrm{N}_{2}$ & 15.6 & 5.13 & $\mathrm{~N}_{2} \mathrm{H}^{+}$ & $\mathrm{CR}$ \\
$\mathrm{CO}$ & 14.0 & 6.15 & $\mathrm{HCO}^{+1}$ & $\mathrm{CR}$ \\
$\mathrm{C}_{2} \mathrm{H}_{2}$ & 11.4 & 6.65 & $\mathrm{C}_{2} \mathrm{H}_{3}^{+}$ & $\mathrm{UV}, \mathrm{CR}$ \\
$\mathrm{HCN}$ & 13.7 & 7.43 & $\mathrm{HCNH}^{+}$ & $\mathrm{UV}, \mathrm{CR}$ \\
\hline $\mathrm{H}_{2} \mathrm{O}$ & 12.6 & 7.22 & $\mathrm{H}_{3} \mathrm{O}^{+2}$ & UV,CR \\
\hline
\end{tabular}

1. Detected in many post-AGB shells.

2. Possible detection in VY CMa.

Table 2 gives the most abundant molecular ions in CSEs in order of increasing proton affinity or $p a$, the binding energy of the proton. The entry for $\mathrm{H}_{2} \mathrm{O}$ is relevant for O-rich CSEs, where it replaces $\mathrm{C}_{2} \mathrm{H}_{2}$ and $\mathrm{HCN}$ as the most abundant molecule after $\mathrm{CO}$. The symbol $\mathrm{CR}$ indicates that the molecular ion is produced by collisional ionization of $\mathrm{H}_{2}$ by a suprathermal particle, usually thought to be a galactic cosmic ray. Ions of smaller $p a$ rapidly lose their proton to ions of larger $p a$ by "proton-transfer" reactions, e.g., $\mathrm{H}_{3}^{+}+\mathrm{CO} \rightarrow \mathrm{HCO}^{+}+$ $\mathrm{H}_{2}$. Molecules with $I P<13.6 \mathrm{eV}$ can be photoionized by the interstellar UV radiation field, and they produce molecular ions by "abstracting" a $\mathrm{H}$ atom from $\mathrm{H}_{2}$, e.g., $\mathrm{C}_{2} \mathrm{H}_{2}^{+}+\mathrm{H}_{2} \rightarrow \mathrm{C}_{2} \mathrm{H}_{3}^{+}+\mathrm{H}$. We see from the table that the ions $\mathrm{H}_{3}^{+}$, $\mathrm{N}_{2} \mathrm{H}^{+}$, and $\mathrm{C}_{2} \mathrm{H}_{3}^{+}$can only be produced from abundant neutrals by suprathermal particles, whereas $\mathrm{C}_{2} \mathrm{H}_{3}^{+}$and $\mathrm{HCNH}^{+}$can also be produced by UV. However, in the neighborhood of the peak abundances of the latter two ions, UV is the main mechanism. Because $\mathrm{H}_{2}$ is the most abundant species in AGB winds with large mass-loss rates, the suprathermal particles mainly create $\mathrm{H}_{2}^{+}$ions that are rapidly transformed into $\mathrm{H}_{3}^{+}$ions by proton transfer to another $\mathrm{H}_{2}$. The $\mathrm{H}_{3}^{+}$ is then transformed into heavier ions, especially $\mathrm{HCNH}^{+}$and $\mathrm{C}_{2} \mathrm{H}_{3}^{+}$. However, these more abundant molecular ions are also difficult to detect (e.g., see Glassgold et al. 1992 for the case of $\mathrm{C}_{2} \mathrm{H}_{3}^{+}$), and most efforts to discover molecular ions have focused on $\mathrm{HCO}^{+}$. In addition to its large dipole moment, this ion has the advantage that it directly measures the suprathermal ionization rate.

Ten years ago Lucas \& Guélin (1988) reported the detection of $\mathrm{HCO}^{+}$with the IRAM 30-m telescope. Lucas \& Guélin (1998) has reported more definitive results at this symposium based on sensitive observations with the IRAM interferometer (Plateau de Bure) and the $30 \mathrm{~m}$. The measurements show that the $J=1-0$ transition has the lineshape expected for an optically-thin, expandingshell and that the observed and laboratory frequencies agree to one part in a million. The emission has also been mapped with the interferometer and is consistent with the partially filled-in shell expected from theory. The line is rather weak, with an average brightness over the line of slightly more than $20 \mathrm{mK}$. The peak abundance of $\mathrm{HCO}^{+}$in IRC +10216 is $\sim 10^{-10}$, and corresponds to a suprathermal ionization rate of $\zeta \approx 2 \times 10^{-18} \mathrm{~s}^{-1}$. This estimate of the ionization 
rate is one order of magnitude smaller than the ionization rate in the solar neighborhood due to (mainly low-energy) galactic cosmic rays, $\zeta_{\mathrm{CR}} \approx 2 \times 10^{-17} \mathrm{~s}^{-1}$.

One explanation of this discrepancy is that the wind of IRC +10216 is magnetized, and that it excludes low-energy cosmic rays in roughly the same way that the solar wind does. ${ }^{2}$ If one uses the modulated cosmic-ray ionization rate measured in the solar neighborhood, then the observed ionization rate in IRC +10216 requires the exclusion of cosmic rays with energies per nucleon $E / A>400 \mathrm{MeV}$. If this is the right explanation, one should be able to explain the numerical value of this cutoff. Although the cosmic ray transport problem is formally solvable, it requires a knowledge of the (unknown) magnetic scattering properties of AGB winds. Alternatively, one could deduce these properties for the wind of IRC +10216 from a wind exclusion calculation.

It is also conceivable that the powerful AGB wind is effective in excluding even higher-energy cosmic rays, in which case the ionization indicated by the presence of $\mathrm{HCO}^{+}$must be generated within the envelope. One possible source is radioactive nuclides dredged up from the stellar interior and carried out into the wind which they ionize by fast $\beta$ particles (Glassgold 1995, 1996b). Two interesting radionuclides synthesized by $\mathrm{AGB}$ stars are ${ }^{26} \mathrm{Al}$ and ${ }^{14} \mathrm{C}$, with mean lifetimes $\tau=1.0 \times 10^{6} \mathrm{yr}$ and $\tau=8100 \mathrm{yr}$, respectively. The decay of ${ }^{26} \mathrm{Al}$ is accompanied by the emission of $\gamma$ rays, with $82 \%$ producing a positron $\left({ }^{26} \mathrm{Al} \rightarrow\right.$ $\left.{ }^{26} \mathrm{Mg}^{*}+\mathrm{e}^{+}+\nu\right)$ and $18 \%$ proceeding by electron capture $\left(\mathrm{e}^{-}+{ }^{26} \mathrm{Al} \rightarrow{ }^{26} \mathrm{Mg}^{*}+\right.$ $\bar{\nu})$. The latter channel gives a few Auger electrons and can be neglected, whereas the positron with mean energy $E_{\beta}\left({ }^{26} \mathrm{Al}\right)=0.66 \mathrm{MeV}$ can produce about 20000 ionizations. AGB winds are too thin to stop the $\gamma$ rays (they interact with the Compton cross-section), and they escape. The decay of ${ }^{14} \mathrm{C}$ is simpler, $\left({ }^{14} \mathrm{C} \rightarrow\right.$ $\left.{ }^{14} \mathrm{~N}+\mathrm{e}^{-}+\bar{\nu}\right)$, with $100 \%$ going to the ground state of ${ }^{14} \mathrm{~N}$ with $E_{\beta}\left({ }^{14} \mathrm{C}\right)=$ $0.047 \mathrm{MeV}$. The two $\beta$ decays heat and ionize the wind in a similar fashion since the physics of the stopping of electrons and positrons is the same for energies above $5 \mathrm{keV}$. However, the depletions of the parent nuclei are different. Whereas about half of the $\mathrm{C}$ is in circumstellar dust particles, much of the $\mathrm{Al}$ may be in solids. A qualitative analysis of the escape of ${ }^{26} \mathrm{Al}$ positrons from dust grains indicates, however, that most of them get into the gas.

The ionization rate $\zeta$ per $\mathrm{H}$ nucleus due to a radioactive decay is (Glassgold 1995)

$$
\zeta=b_{\beta} \frac{1}{\tau} \mathcal{R} x \frac{E_{\beta}}{e_{\text {ion }}} \exp (-r / V \tau),
$$

where $b_{\beta}$ is the branching ratio for $\beta$-emission $\left(0.82\right.$ for ${ }^{26} \mathrm{Al}$ and 1.00 for $\left.{ }^{14} \mathrm{C}\right)$, $e_{\text {ion }}=35 \mathrm{eV}$ is the mean energy to create an ion pair in a predominantly $\mathrm{H}_{2} / \mathrm{He}$ gas, $\mathcal{R}$ is the abundance ratio of a radioactive to a stable nuclide, the latter with absolute abundance $x$. For the cases of interest here, $r<V \tau$, and the rates are:

$$
\zeta_{26} \approx 1.4 \times 10^{-15} \mathcal{R}_{26} \mathrm{~s}^{-1} \quad \zeta_{14} \approx 1.0 \times 10^{-12} \mathcal{R}_{14} \mathrm{~s}^{-1}
$$

with $x\left({ }^{27} \mathrm{Al}\right)=3 \times 10^{-6}($ solar $)$ and $x\left({ }^{12} \mathrm{C}\right)=4.5 \times 10^{-4}$.

A small upper limit to the abundance of ${ }^{14} \mathrm{C}$ in $\mathrm{IRC}+10216$ has been obtained by a sensitive search for ${ }^{14} \mathrm{CO}$ (Forestini et al. 1997), with the result

\footnotetext{
${ }^{2}$ No one has ever solved the cosmic ray transport problem for any wind other than the Sun's.
} 
$\mathcal{R}_{14}<1.5 \times 10^{-5}$. Using Eq. (1), this yields $\zeta_{14}<1.5 \times 10^{-17} \mathrm{~s}^{-1}$ for IRC +10216 . This upper limit is significantly larger than the ionization rate determined from the observations of $\mathrm{HCO}^{+}$. Forestini et al. (1997) consider the prospects for detecting ${ }^{14} \mathrm{CO}$ to be dim. The attempts by Guélin et al. (1995) to detect ${ }^{26} \mathrm{Al}$ molecules in the CSE of IRC +10216 have not been definitive because of confusion and blending with other lines. They quote an upper limit, $\mathcal{R}_{26}<0.04$, based on observations of the $J=7-6$ line of ${ }^{26} \mathrm{AlF}$ (assuming that all $\mathrm{Al}$ is in the gas phase). The most recent nucleosynthetic calculations for an intermediatemass AGB star $\left(M=4.5 M_{\odot}\right)$ give $\mathcal{R}_{26} \sim 10^{-3}$ (Lattanzio \& Forestini 1998). If we substitute this estimate into Eq. (1), we find $\zeta_{26} \sim 1.4 \times 10^{-18} \mathrm{~s}^{-1}$, the same order as the value deduced from the observations of $\mathrm{HCO}^{+}$in IRC +10216 . Amari et al. (1998) report measurements of $\mathcal{R}_{26}$ for "mainstream" presolar $\mathrm{SiC}$ grains $\left({ }^{12} \mathrm{C} /{ }^{13} \mathrm{C}=30-80\right)$ that are in the range $10^{-4}-10^{-3}$. The upper part of this range is the same order of magnitude as predicted by nucleosynthesis; it is also consistent with the ionization rate determined from $\mathrm{HCO}^{+}$.

We therefore regard live ${ }^{26} \mathrm{Al}$ as a viable explanation for the level of suprathermal ionization observed in IRC +10216 . It would be reassuring to detect ${ }^{26} \mathrm{Al}$ molecules, as attempted by Guélin et al. (1995), and to detect other molecular ions such as $\mathrm{C}_{2} \mathrm{H}_{3}^{+}$and $\mathrm{HCNH}^{+}$.

\section{Discussion}

Although the photochemical model is successful in providing a qualitative understanding of many aspects of AGB wind chemistry, there are problems in making it more quantitative. Some of these issues have been mentioned in Sect. 2.5, and here we address two important observational issues, the clumpiness and the size of the photochemical shells.

The IRAM observations (e.g., Lucas \& Guélin 1998) show that the outer shell distributions are fragmented into what appears to be large clumps, (typically $5^{\prime \prime} \times 10^{\prime \prime}$ ) in IRC +10216 . The fact that they are common to several species implies that they are density enhancements. If so, they potentially can affect the dynamics, radiative transfer, and physical processes, including the chemistry. The origin as well as the detailed nature of these clumps are unknown, but they could be studied directly by $\mathrm{mm}$ interferometry. For example, it would be of considerable interest to map the clumps in a density-sensitive line such as ${ }^{13} \mathrm{CO}$. To obtain a deeper theoretical understanding, one needs more sophisticated modeling that incorporates a richer dynamical and physical treatment than attempted so far.

The IRAM observations (e.g., Lucas \& Guélin 1998) also show that many of the shells have same radii $\left(\approx 15^{\prime \prime}\right.$, or $3000 \mathrm{AU}$ at $\left.200 \mathrm{pc}\right)$. There are some exceptions that result from interior production, e.g., $\mathrm{SiC}_{2}, \mathrm{HNC}, \mathrm{HCO}^{+}$, or from weak photo-destruction that yields large shells, e.g., CN. In the current version of the photochemical model, as described in Sect. 1 and Sect. 2, the shell separation arises because the dynamical (expansion) and chemical timescales are about the same in the shells, $\approx 300 \mathrm{yr}$. Thus one simple way of reducing the differences in shell radii is to reduce the chemical time by increasing the density. This idea is consistent with clumping. Like the treatment of clumps, however, a sound understanding of this issue requires a new dynamical chemistry of CSEs. 
Acknowledgments. The research on which this survey is based is funded in part by the National Science Foundation.

\section{References}

Amari S., Nittler L.R., Zinner E., Lewis R.S., 1998, poster contribution, this conference

Cherchneff I., Glassgold A.E., Mamon G.A., 1993a, ApJ 410, 188 (CGM)

Cherchneff I., Glassgold A.E., Mamon G.A., 1993b, ApJ 419, L41

Forestini M., Guélin M., Cernicharo J., 1997, A\&A 317, 883

Gensheimer P.D., Likkel L., Snyder L.E., 1995, ApJ 439, 445

Glassgold A.E., 1995, ApJ 438, L111

Glassgold A.E., 1996a, ARA\&A 34, 241

Glassgold A.E., 1996b, in Amazing Light, R. Chiao (ed.), Springer, New York, p. 277

Glassgold A.E., Lucas R., Omont A., 1986, A\&A 157, 35

Glassgold A.E., Omont A., Guélin M., 1992, ApJ 396, 115

Glassgold A.E., Mamon G.A., 1993, in Chemistry and Spectroscopy of Interstellar Molecules, D.K. Bohme, E. Herbst, N. Kaifu and S. Saito (eds.), Tokyo, p. 263

Groenewegen M.A.T., van der Veen W.E.C.J., Matthews H.E., 1998, A\&A 338, 491

Guélin M., Forestini M., Valiron P., Ziurys L.M., Anderson M.A., Cernicharo J., Kahane C., 1995, A\&A 297, 183

Howe D.A., Millar T.J., 1990, MNRAS 244, 444

Keady J.J., Ridgway S., 1993, ApJ 406, 199

Lattanzio J., Forestini M., 1998, this volume

Lodders K., Fegley B., 1995, Meteoritics 30, 661

Lucas R., 1994, in Astrometry with Millimeter \& Submillimeter Interferometry, M. Ishiguro \& W.J. Welsh (eds.), ASP, San Francisco, p. 135

Lucas R., Guélin M., 1988, in Submillimeter and Millimeter Astronomy, W.B. Burton \& R.D. Wolstencroft (eds.), Reidel, Dordrecht, p. 97

Lucas R., Guélin M., 1988, this volume MacKay D.D.S., Charnley S.B., 1998, MNRAS in press

Mamon G.A., Glassgold A.E., Huggins P.J., 1988, ApJ 328, 797

Millar T.J., Herbst E., 1994, A\&A 288, 561

Willacy K., Cherchneff I., 1997, A\&A 330, 676

Willson L.A., Struck C., Bowen G.H., 1997, in Cosmic Winds \& The Heliosphere, J.R. Jokipii, P.C. Sonnett and M.S. Giampapa (eds.), Tucson, p. 155

Zuckerman B., Dyck H.M., Claussen M., 1986, ApJ 304, 401 